\title{
Recapitulating the Bayesian framework for neurosurgical outpatient care and a cost-benefit analysis of telemedicine for socioeconomically disadvantaged patients in the Philippines during the pandemic
}

\author{
Kevin Paul Ferraris, MD, MBA, ${ }^{1}$ Jared Paul Golidtum, MD, MPM,, Brian Karlo W. Zuñiga, BS, ${ }^{2}$ \\ Maria Cristina G. Bautista, PhD, ${ }^{3}$ Jose Carlos Alcazaren, MD, ${ }^{1}$ Kenny Seng, MD, ${ }^{1,4}$ and \\ Joseph Erroll Navarro, MD'
}

${ }^{1}$ Section of Neurosurgery, Department of Surgery, Jose R. Reyes Memorial Medical Center, Manila; ${ }^{2}$ Health Sciences Program, School of Science and Engineering, Loyola Schools, Ateneo de Manila University, Quezon City; ${ }^{3}$ Department of Economics, Finance and Accounting, Graduate School of Business, Professional Schools, Ateneo de Manila University, Makati; and ${ }^{4}$ Division of Neurosurgery, Department of Neurosciences, University of the Philippines-Philippine General Hospital, University of the Philippines College of Medicine, Manila, Philippines

OBJECTIVE In the Philippines during recent months, a neurosurgical center that caters primarily to socioeconomically disadvantaged patients has encountered unprecedented changes in practice patterns brought about by the COVID-19 pandemic. In particular, the usual task of outpatient care has shifted to the telemedicine format, bringing along all of its attendant advantages and gargantuan challenges. The authors sought to determine the responsiveness of this telemedicine setup to the needs of their disadvantaged patients and explored the application of Bayesian inference to enhance the use of teleconsultation in daily clinical decision-making.

METHODS The authors used the following methods to assess the telemedicine setup used in a low-resource setting during the pandemic: 1) a cross-sectional survey of patients who participated in a medical consultation via telemedicine during the 16-week period from March 16, 2020, to July 15, 2020; 2) a cost-benefit analysis of the use of telemedicine by patients; and 3) a case illustration of a Bayesian approach application unique to the teleconsultation scenario.

RESULTS Of the 272 patient beneficiaries of telemedicine in a 16 -week period, 57 responded to the survey. The survey responses regarding neurosurgical outpatient care through telemedicine yielded high ratings of utility for the patients and their caregivers. According to $64 \%$ of respondents, the affordability of the telemedicine setup also prevented them from borrowing money from others, among other adverse life events prevented. There were realized financial gains on the part of the patients in terms of cost savings and protection from further impoverishment. The benefit-cost ratio was 3.51 for the patients, signifying that the benefits outweighed the costs. An actual teleconsultation case vignette was reported that is meant to be instructive and contributory to the preparedness of the neurosurgeon on the provider end of the service delivery.

CONCLUSIONS Telemedicine holds promise as a viable and safe method for health service delivery during the pandemic. In the setting of a health system that is continually challenged by shortages of resources, this study shows that an effective telemedicine setup can come with high benefit-cost ratios and quality of care, along with the assurance of patient satisfaction. The potential for high-quality care can be enhanced by the inclusion of the Bayesian framework to the basic toolkit of remote clinical assessment. When confronted with choices in terms of differential diagnosis and tests, the rigor of a simple application of the Bayesian framework can minimize costs arising from uncertainties.

https://thejns.org/doi/abs/10.3171/2020.9.FOCUS20695

KEYWORDS pandemic; telemedicine; cost-benefit analysis; Bayesian framework; clinical decision-making

ABBREVIATIONS BCR = benefit-cost ratio; $\mathrm{CBA}$ = cost-benefit analysis; $\mathrm{SAH}$ = subarachnoid hemorrhage; $\mathrm{TBM}$ = tuberculous meningitis.

SUBMITTED July 31, 2020. ACCEPTED September 18, 2020.

INCLUDE WHEN CITING DOI: 10.3171/2020.9.FOCUS20695. 
$\mathrm{T}$ HE effect of the COVID-19 pandemic is felt not only by those with coronavirus disease, but also by those who are suffering from any illness and whose access to care is curtailed by diminished healthcare availability that is exacerbated by excess demand for the various services of the health system. This situation has particularly affected the continuity of care of patients needing neurosurgical consultations and operations. ${ }^{1}$ In the Philippines, access to neurosurgical specialty care among the poor segments of the population is found wanting, owing to the presence of few public hospitals with comprehensive neurosurgery training centers in the capital region. ${ }^{2}$ During the unfolding pandemic, the national government has enforced a strict community quarantine that has restricted travel and influenced hospital policies across the country. Difficult decisions abound on how to continue neurosurgical diagnosis and treatment functions at this time. The use of telemedicine as applied to neurosurgery ${ }^{3-7}$ during this pandemic has seen a dramatic rise and resurgence of interest and is currently encouraged in local guidelines. ${ }^{8,9}$ During these extraordinary times, enhancing the telemedicine system is recommended to optimize the care of patients.

\section{Methods}

The aim of this study was to assess the responsiveness of a current telemedicine setup during the COVID-19 pandemic by using the following methods: 1) a utility and expenditure survey of patient telemedicine users, 2) a costbenefit analysis (CBA) from the perspective of the patient, and 3) a case illustration of a Bayesian approach application unique to the teleconsultation scenario. This study was approved by the medical center institutional review board.

\section{Provider Setting and Socioeconomic Context}

Jose R. Reyes Memorial Medical Center is a multispecialty, government-funded hospital located in Manila that primarily caters to the socioeconomically disadvantaged catchment population all over the Philippine archipelago. Over the years, the Center's Section of Neurosurgery has performed 600 to 800 wide-ranging emergency and subspecialty elective operations annually. While emergency neurosurgical operations are allowed to continue during the pandemic, the in-person outpatient consultation and scheduling of elective surgeries have come to a halt. Providing comprehensive outpatient neurosurgical care in the midst of the pandemic is a challenging proposition. In many areas of the country, particularly in the national capital, the enhanced community quarantine suspended all public mass transportation and severely restricted the movement of people. The telemedicine format of health service delivery partially fills the current gap in healthcare availability.

\section{Telemedicine Protocol}

Since mid-March 2020, outpatient teleconsultation with neurosurgical residents has been made available to neurosurgical patients primarily via online text messaging and secondarily via video calls or phone calls whenever deemed necessary. Freely accessible online modules and learning materials related to telemedicine were used by the telehealth providers for supplementary training. According to a local telemedicine manual by Isip-Tan and colleagues, ${ }^{9}$ a messaging function can be an adequate mode of telehealth given that the majority of Filipinos, who, regardless of socioeconomic status, often have the means for the minimum internet access ${ }^{10}$ needed to maintain a social media account, ${ }^{11}$ with the Facebook platform as the popular choice..$^{12,13}$ The chat messaging feature Facebook Messenger appears to be an attractive tool for doctor-patient communication as a bridging and improvised telehealth platform solely for the purpose of increasing access to care. The legal milieu surrounding this use of internet platforms has been relaxed, which appears to be justified during this time of the pandemic.,14,15 Through this novel protocol-driven neurosurgical service brought about by demand (Fig. 1), the basic outpatient functions of triaging, follow-up care, symptom assessment, medication adjustment, and other functions can be conducted online.

\section{Patient Survey of Telemedicine Utility and Expenditure Patterns}

For a minimum calculated sample size of 48 , a crosssectional survey of the patients who participated in a medical consultation via telemedicine during the 16-week period from March 16, 2020, to July 15, 2020, was done. The survey questions addressed the participants' overall patient experience, asking questions on the elements for achieving quality in telemedicine as determined by the Institute of Medicine, ${ }^{16}$ specifically efficiency, adequacy, timeliness, and satisfaction. The survey form included questions in both English and the native language, with an anchoring sample scenario per the aforementioned elements, while the answers consisted of ordinal choices along a 10-point Likert scale. Expenditure patterns of the patients and their caregivers were collected in the same survey form and obtained via open-ended questions for interval-ratio answers. The monetary expenses surrounding the use of telemedicine were interpreted in terms of catastrophic and impoverishing expenditures. ${ }^{17-19}$

\section{Cost-Benefit Analysis}

This health-related CBA assumed the economic perspective of the patient ${ }^{20}$ The consolidated costs comprised the direct costs incurred by the patient for utilizing the telemedicine intervention plus the monetized indirect costs of economic productivity loss, while the consolidated benefits consisted of the averted travel cost plus the monetized indirect benefit of lost productivity due to the alternative of an in-person clinic visit. The benefit-cost ratio (BCR) was calculated. To determine whether or not the monetary benefits of an intervention outweigh its costs, the following formula was used: $\mathrm{BCR}=$ consolidated benefits/consolidated costs. A value $>1$ means that benefits outweigh costs, and the evaluated health intervention is beneficial.

\section{Statistical Analysis}

Descriptive statistics were used to summarize the general and clinical characteristics of the respondents. Frequency and proportion were used for nominal variables, median and range for ordinal variables, and mean and standard 


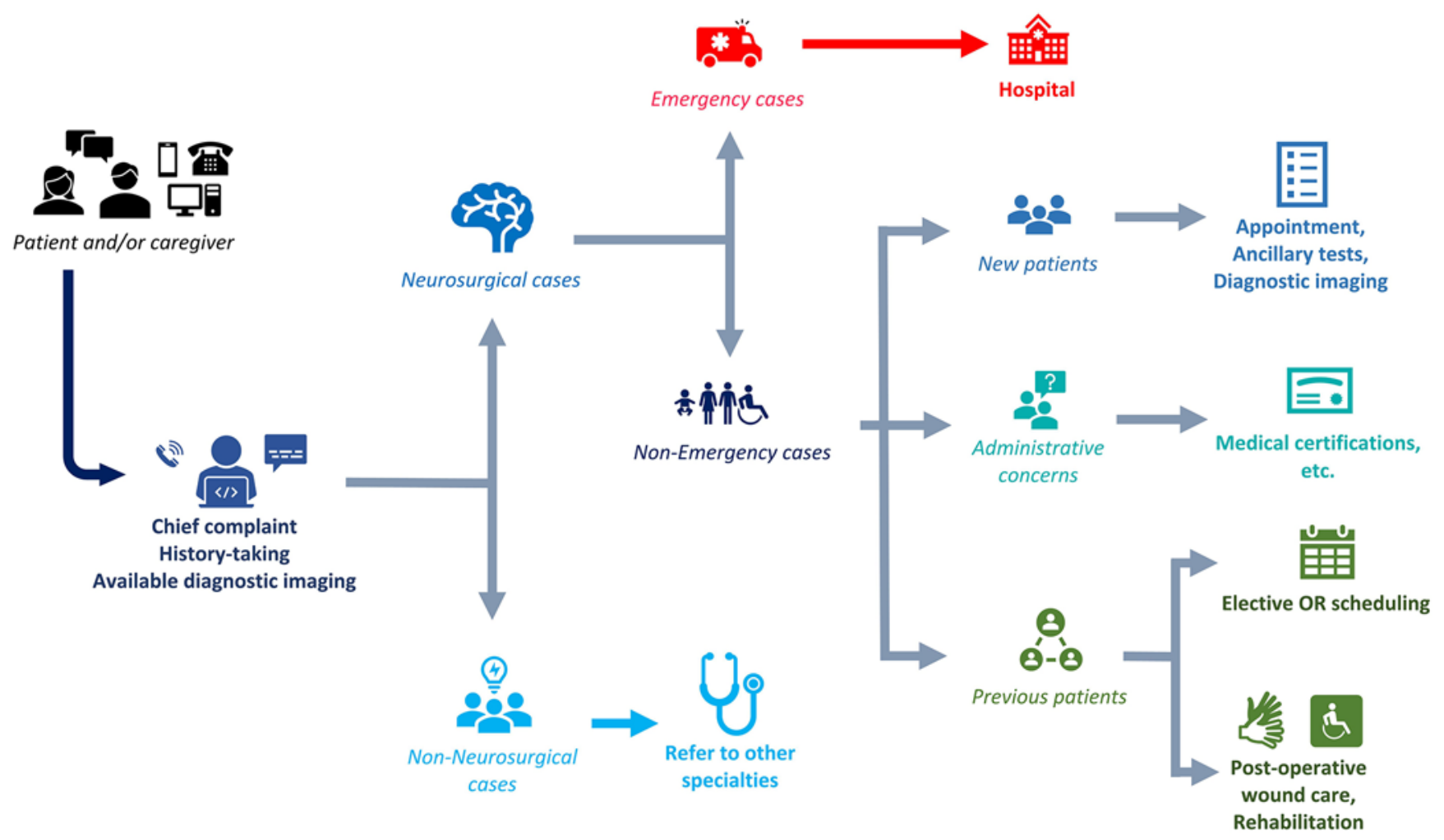

FIG. 1. Process flow and algorithm of the telemedicine setup used at Jose R. Reyes Memorial Medical Center, Manila, Philippines, during the time of the COVID-19 pandemic.

deviation for interval-ratio variables. The null hypothesis was rejected at the 0.05 level of significance. Stata software version 15.0 (StataCorp) was used for data analysis.

\section{Case Illustration of Bayesian Analysis}

Work in Bayesian statistical models has long complemented the practice of evidence-based medicine, but its applications in daily clinical decision-making appear elusive. ${ }^{21}$ We present a case study that offers some lessons on the application of the Bayesian framework to the clinical encounter conducted in the telemedicine platform.

\section{Results and Discussion}

There were 272 beneficiaries who used telemedicine during the study period, and 57 responded to the survey. The mean age of the respondents was $39 \pm 13$ years (Table 1). The majority of respondents were male (57\%), considered their places of residence as far from the neurosurgi$\mathrm{cal} /$ medical center (77\%), and had a monthly income at or below 10,000 Philippine pesos (P10,000; 67\%). The observed frequencies of consultation types significantly differed from expected proportions $(\mathrm{p}<0.001)$, with the greatest proportion of the consultations classified as neurosurgical cases. Likewise, for the neurosurgical cases deemed amenable to teleconsultation, the distribution of cases in terms of being a new patient, previous patient as follow-up, or previous patient as postoperative also significantly differed from each other $(\mathrm{p}<0.001)$.

The majority of respondents provided positive ratings for the efficiency, adequacy, timeliness, and overall satisfaction of their telemedicine experience (Fig. 2). Fewer than $2 \%$ of respondents gave a score of less than 5 out of 10 for either efficiency or adequacy.

In the absence of teleconsultation, more than half of the respondents reported that they would need to borrow money (64\%) for the alternative of in-person consultation in the outpatient clinic. Roughly 4 in 10 each said they would seek a babysitter or may lose income. About $11 \%$ each indicated that they needed to interrupt their children's education, were forced to seek charity, or resorted to pawning valuable possessions (Table 2).

Telemedicine averted catastrophic expenditures ${ }^{22}$ for $32 \%$ (95\% CI 20\%-46\%) of respondent patients and their families had they used the alternative of in-person consultation.

As for impoverishing expenditures, ${ }^{23}$ using the value of P10,756 as the monthly poverty threshold for a family of five, ${ }^{24} 72 \%$ (95\% CI 58\%-83\%) of the respondents avoided further impoverishment due to the telemedicine setup (Table 2). Among the impoverished, $42 \%$ reported having no income during the pandemic and were already living below the poverty line to begin with.

Total costs from combined telecommunication charges and lost productivity of the patient and caregiver were estimated at P430 per patient consult and P24,508 for all samples. The total benefits to be gained per patient and by all patients were P1938 and P110,461, respectively. Net benefits per patient and for all patients were P1508 and P85,953, respectively. The calculated BCR was 3.51 (Table 3 ). 
TABLE 1. Telemedicine patient beneficiary and survey respondent characteristics

\begin{tabular}{lc}
\hline & Value \\
\hline Telemedicine beneficiaries $(\mathrm{n}=272)$ & $188(69.1)$ \\
\hline Adult & $84(30.9)$ \\
\hline Pediatric & $7(2.6)$ \\
\hline Categories during teleconsult & $34(12.5)$ \\
\hline Administrative query & $24(8.8)$ \\
\hline Triaged to emergency department & \\
\hline Referred to other specialties* & $39(14.4)$ \\
\hline Neurosurgical case amenable to teleconsultation & $144(52.9)$ \\
\hline New patient & $24(8.8)$ \\
\hline Previous patient as follow-up & $57(20.9)$ \\
\hline Previous patient as postop & $39 \pm 13.01$ \\
\hline Survey respondents $(n=57)$ & $32(56.1)$ \\
\hline Age, yrs & $25(43.9)$ \\
\hline Sex & \\
\hline Male & $12(22.6)$ \\
\hline Female & $41(77.4)$ \\
\hline Patient-perceived distance from center $(n=53)$ & $38(66.7)$ \\
\hline Near & $17(29.8)$ \\
\hline Far & $2(3.5)$ \\
\hline Monthly income, Philippine pesos $(n=57)$ & \\
\hline$\leq 10,000$ & \\
\hline $10,001-30,000$ & \\
\hline$>30,000$ & \\
\hline
\end{tabular}

Values are presented as the number (\%) of telemedicine beneficiaries or survey respondents or as the mean \pm SD.

${ }^{*}$ Not mutually exclusive with other categories.

\section{Intangible Benefits of Telemedicine in Low-Income Settings}

The adoption of telemedicine by the patients in our setting appears to be most attractive for use by neurosurgical patients needing follow-up. Telemedicine helps to decrease unnecessary hospital visits by allowing the physician to provide patient reassurance for a minor concern. This setup also allows urgent concerns to be addressed as soon as possible through a video call, phone call, or text message, whereas nonurgent concerns are replied to within reasonable time frames that are free from the constraints of a physical clinic schedule. In terms of the aspect of adequacy, respondents experience a degree of freedom in being able to ask questions without the time constraints of an in-person consultation. Furthermore, the triaging function of telemedicine grants timely medical advice to patients who need to be evaluated emergently. Given that the majority of the respondents perceive the medical center to be far from their homes, notwithstanding the unavailability of transportation during the pandemic, telemedicine offers a degree of convenience to socioeconomically disadvantaged patients. In cases for which a patient needs a neurosurgical evaluation but is admitted to a hospital with only lower levels of care, telemedicine helps decrease unnecessary transfers and also allows care management decisions to be addressed in a timely manner (e.g., providing prehospital bundles-of-care advice or administering physician-ordered osmotic diuretic medication for a neurosurgical emergency)-features that can have a positive impact on long-term outcomes of patients in our low-resource catchment areas. Further studies are needed to identify the potential benefits of telemedicine in addressing the perennial problems of neurosurgical care in low-income countries, including loss to follow-up and difficulties in long-term outcome assessment. ${ }^{25}$

\section{Cost Savings and Financial Risk Protection Associated With Telemedicine}

In addition to the intangible benefits, our telemedicine setup appears to be a cost-beneficial intervention. This benefit can improve the health-seeking behavior of patients and their families, given that many of them reported the possibility of adverse life events were it not for the cost savings afforded by telemedicine. This can translate to increased adoption of telemedicine and, consequently, utilization of various aspects of comprehensive care by neurosurgical patients (e.g., rehabilitation plans for neurotrauma and reminder tools for adjuvant therapies) and for time-dependent follow-up care usually done by neurosurgeons (e.g., monitoring of children after shunt placement, and assessment of delayed complications of neurotrauma, such as CSF leak). For impoverished patients, costs constitute an important consideration and even a barrier to proper care-but the telemedicine setup is deemed responsive to the needs of our patients because protection from financial risk, including impoverishing and catastrophic expenditures, is also afforded. Therefore, it behooves the healthcare providers to continuously rethink which aspects of neurosurgical care in low-income settings can be better aided by telemedicine.

In cost-related studies by Thakar and colleagues ${ }^{26}$ and Dadlani and colleagues ${ }^{27}$ telemedicine for neurosurgical outpatient follow-up care by patients in socioeconomically disadvantaged backgrounds is similarly found to be promising and cost-effective. However, there are inherent limitations to cost-related studies, ${ }^{28}$ as well as to our study, which include the heterogeneous clinical profiles of our patients, the limited study duration, and the unaccountedfor confounding factors. Additionally, we did not evaluate the clinical efficacy of the particular kind of care delivered through telemedicine, as similarly pointed out in a study by Ahmed and colleagues, ${ }^{29}$ which was also approached from the patient's perspective for CBA. However, the results of our CBA further highlight the magnitude of the pandemic-related need for access to neurosurgical outpatient care similarly found in contemporary studies. ${ }^{3-7}$

\section{Telemedicine as a Toolkit to Solve the Patient's Problems}

Ultimately, telemedicine mitigates the gap in access to neurosurgical care, with a substantial benefit to lowincome neurosurgical patients who also bear the difficulty of travel and the associated incidental costs. ${ }^{26,27,30}$ However, the effective and outcome-oriented delivery of care to patients with neurological complaints introduces particular challenges to telehealth providers. The usual process of clinical encounters-from history taking and diagno- 


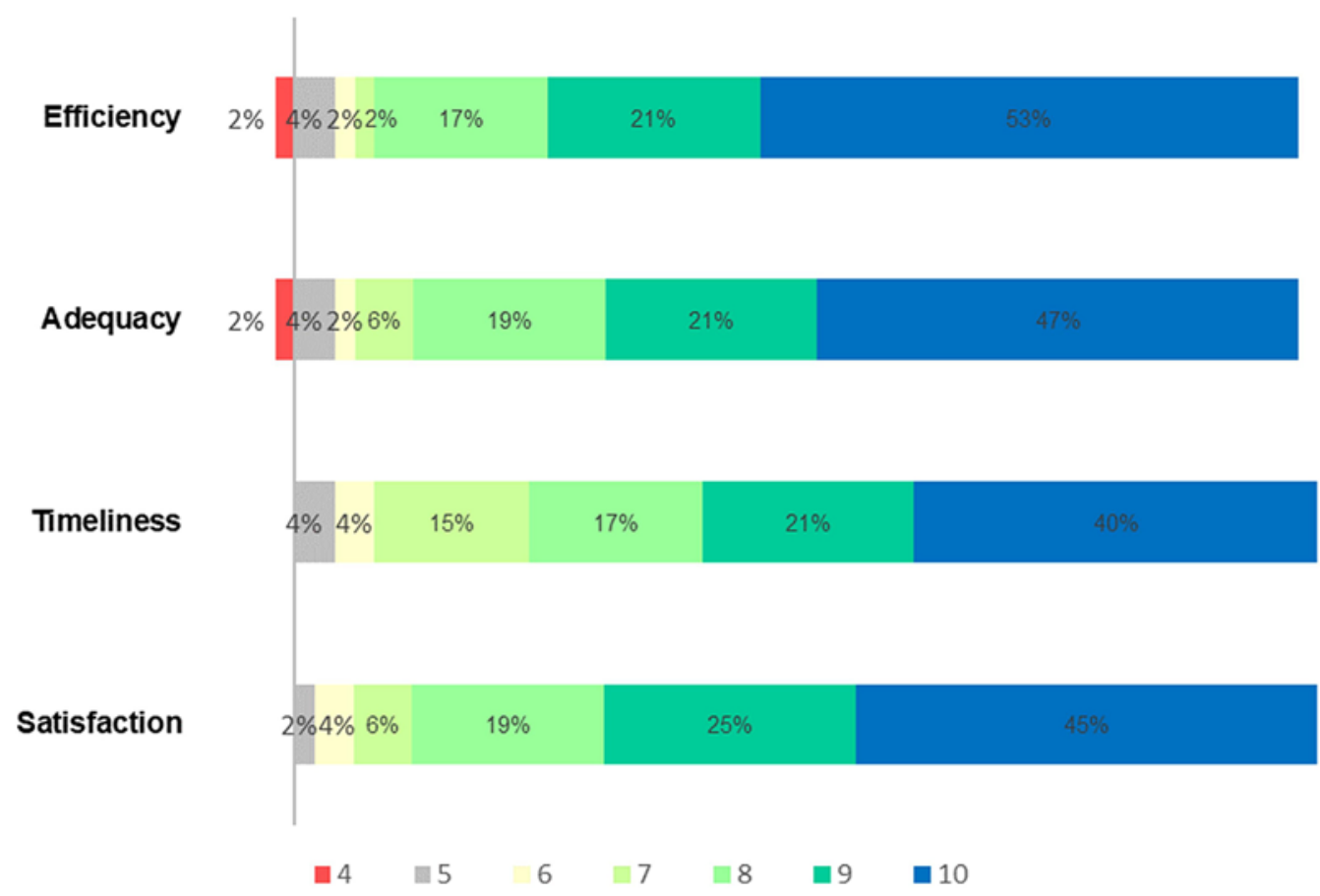

FIG. 2. Perceptions reported by patient questionnaire respondents regarding the quality of their telemedicine experience on a scale of $1-10$, with 10 being the highest positive rating.

sis to treatment and prognostication-is subject to unique constraints and limitations that are inherent to the telemedicine platform and the macro-level changes of health service delivery brought about by the pandemic. Current practice patterns require certain nuances in the approach to the patient and in clinical reasoning.

\section{Case Vignette of Teleconsultation}

Consider the following case of an actual telemedicine consultation. A 46-year-old man was brought to a local district hospital due to severe headache. The hospital does not have subspecialty services. When advised of the need for a "neuroconsult" and transfer to another hospital with higher-level care, the patient's wife (respondent) participated in an online telemedicine consultation. First, the physician determined that the patient's symptoms started 10 days prior, with the headache characterized as throbbing, located at the right temporal and occipital regions, occasionally radiating to the left side, graded subjectively at $8 / 10$, and slightly relieved by intake of etoricoxib once daily. The reason for the emergency consultation at the district hospital was the increase in severity of the patient's headache to $10 / 10$. The following dialogue is the continuation of an adapted excerpt of the conversation via telemedicine:

Doctor: What happened leading to your husband's admission? Respondent: He's become hysterical due to the pain. We can no longer stand him screaming and shouting due to the severe headache.

Doctor: Any aggravating factors?

Respondent: Any movement of his head.
Doctor: Did he have this kind of headache prior to this?

Respondent: No.

Doctor: Does he have comorbids? Any history of previous hospitalizations and/or surgeries? History of trauma or fall?

Respondent: None. We all think he is quite healthy previously.

Doctor: Did he have other symptoms: loss of consciousness, seizure, vomiting, weakness or numbness of one part of the body, or visual changes?

Respondent: He had vomiting of his previously ingested food on our way to the hospital. But he had none of those other things you mentioned.

Doctor: Did you consult a doctor at any time during the 10-day duration of his persistent headache?

Respondent: Yes. During the 6th day of headache, he was seen by a general practitioner who requested a noncontrast head CT scan. We had it done right away.

The respondent then proceeded to pull out and send a photo of a paper bearing the official result of the CT, which was essentially normal. There was no available photo of the actual CT.

Without the benefit of a physical and a neurological examination, the neurosurgeon proceeded with ruminating on the patient's condition for definitive management. The list of differential diagnosis (in no particular order of likelihood) may include migraine, cluster headache, tension-type headache, aneurysmal subarachnoid hemorrhage (SAH), hypertensive encephalopathy, venous sinus thrombosis, dural arteriovenous fistula, tumor-related hemorrhage, sinusitis, and temporal arteritis.

\section{Bayes' Theorem in Incomplete History Taking}

In its basic form, Bayes' theorem allows the calcula- 
TABLE 2. Adverse life events and financial risk averted by telemedicine option $(n=57)$

\begin{tabular}{lc}
\hline \multicolumn{1}{c}{ Adverse Life Event } & Value \\
\hline Needed to borrow money & $35(61.4)$ \\
\hline Forced to seek babysitter & $22(38.6)$ \\
\hline Loss of job/income & $23(40.4)$ \\
\hline Discontinuation of child education & $6(10.5)$ \\
\hline Forced to accept charity & $6(10.5)$ \\
\hline Needed to pawn possessed valuables & $6(10.5)$ \\
\hline Catastrophic expenditure* $^{*}$ & $41(72 \%)[58-83 \%]$ \\
\hline Impoverishing expenditure $\dagger$ & $21(37 \%)[24-51 \%]$ \\
\hline
\end{tabular}

Values are presented as number of respondents (\%) $[95 \% \mathrm{Cl}$.

* Catastrophic expenditure is defined as "a household's financial contribution to the health system exceeds $40 \%$ of the income remaining after subsistence needs have been met."22

$\dagger$ Impoverishing expenditure is defined as "expenditures that leave household non-medical consumption below or further below subsistence levels, as defined by a poverty line."23

tion of the probability of an event-such as the presence of a disease-given some new piece of information or evidence that has been obtained and that contributes to the likelihood of that event. ${ }^{21,31-34}$ In the case we present, the neurosurgeon-telehealth provider noted the rather high prevalence of stroke in the age group of the patient as well as the distinguishing feature of severe headache in $\mathrm{SAH} .{ }^{35}$ The patient's previous headaches of lesser severity can be attributed to sentinel headaches from a premonitory minor leak of an intracranial aneurysm, ${ }^{36}$ thus giving the diagnosis of a ruptured aneurysm a high prior probability. Through a series of comprehensive questions from which the helpful answers serve to add new information and increase likelihood of a certain differential diagnosis, a particular disease considered likely by the neurosurgeon either ascends or descends in the posterior probability, thus allowing the neurosurgeon to rule in or rule out some of the possible diseases or conditions.

The novice or the hurried physician may not able to perform a comprehensive history taking that would facilitate the determination of increased likelihood of a certain diagnosis. In the aforementioned dialogue, the presence or absence of fever was not ascertained from the respondent. The effect of this failure to elicit a pertinent symptom or sign is the inadvertent nonconsideration of inflammatory or infectious disease entities. In the actual case example, the telehealth provider asked if the patient had a fever, to which the respondent's answer was: "I don't know... but his forehead was warm to touch." A careful approach would be to take this response equivocally ${ }^{37}$ and invoke the prior probability of another likely differential by remembering the endemicity and high prevalence ${ }^{38}$ of a subacute form of central nervous system infection-tuberculous meningitis (TBM) - which also features headache of a few days' duration, low-grade fever, and behavioral changes, among its other protean presentations. ${ }^{39,40}$

\section{Bayes' Theorem in Clinical Decision-Making}

In considering the next course of action, a primary
TABLE 3. Costs and benefits of telemedicine consults

\begin{tabular}{lrc}
\hline & Per Patient & All Patients $(\mathrm{n}=57)$ \\
\hline Costs & & \\
\hline Telecommunication charges & 156.25 & 8906.25 \\
\hline Productivity loss & 273.71 & $15,601.47$ \\
\hline Total costs & 429.96 & $24,507.72$ \\
\hline Benefits & \\
\hline Transportation cost savings & 1053.67 & $60,059.19$ \\
\hline Averted productivity loss & 884.23 & $50,401.11$ \\
\hline Total benefits & 1937.90 & $110,460.30$ \\
\hline Net benefit & 1507.94 & $85,952.58$ \\
\hline BCR & 3.51 & 3.51 \\
\hline
\end{tabular}

All values are in Philippine pesos unless otherwise indicated.

working impression would revolve around choosing between SAH and TBM. For these two conditions, timely management will significantly alter the outcome and prognosis. Still ambiguous at this point, each differential is assigned a prior or pretest probability of $50 \%$. Using the nomogram version of Bayes' theorem, ${ }^{21}$ there is a need to be less uncertain and factor in the likelihood that a test can contribute and might better increase the posterior or posttest probability of one disease over the other. Since it is highly possible that tests might lead to a change in management strategies, the question arises as to which test is most appropriate.

Can a life-threatening ruptured intracranial aneurysm be ruled out? Can a repeat CT be reconsidered, given that the chance of detecting a thin SAH diminishes by $50 \%$ after 7 days? ${ }^{36}$ Should an angiographic study be requested upfront or, given the uncertainty that the patient has clearcut $\mathrm{SAH}$, is it reasonable to be reticent in requesting any costly imaging tests? The patient would be better off undergoing a single test that could adequately rule in or rule out both SAH and TBM. A simple lumbar tap for CSF analysis appears appropriate, because it contributes the likelihood ratios of 4.03 for detecting xanthochromia in $\mathrm{SAH}^{41}$ and 1.89 for detecting the CSF parameters consistent with TBM..$^{42}$ By plotting the pretest probabilities and likelihood ratios into the Bayes' nomogram, estimates of the respective posttest probabilities of SAH and TBM in the patient are made. Similar to a lengthy formula-based calculation, the nomograms yield the posttest probabilities of approximately 65\% for TBM (Fig. 3A) and approximately $80 \%$ for SAH (Fig. 3B).

Going back to the actual case, the patient was eventually transferred to a multispecialty hospital and additional ancillary studies were done. The CSF analysis after the lumbar tap revealed minimal hypoglycorrhachia and pleocytosis with lymphocytic predominance. The patient's chest radiograph showed suspicious apical densities. The attending physician was able to elicit an additional feature from a repeat history taking: unintentional weight loss during the past year. Given the benefit of these new data, the pretest probabilities can be adjusted in retrospect. TBM may now present a greater degree of prior probability of $60 \%$, compared with $40 \%$ for SAH (Fig. 3C and D). 


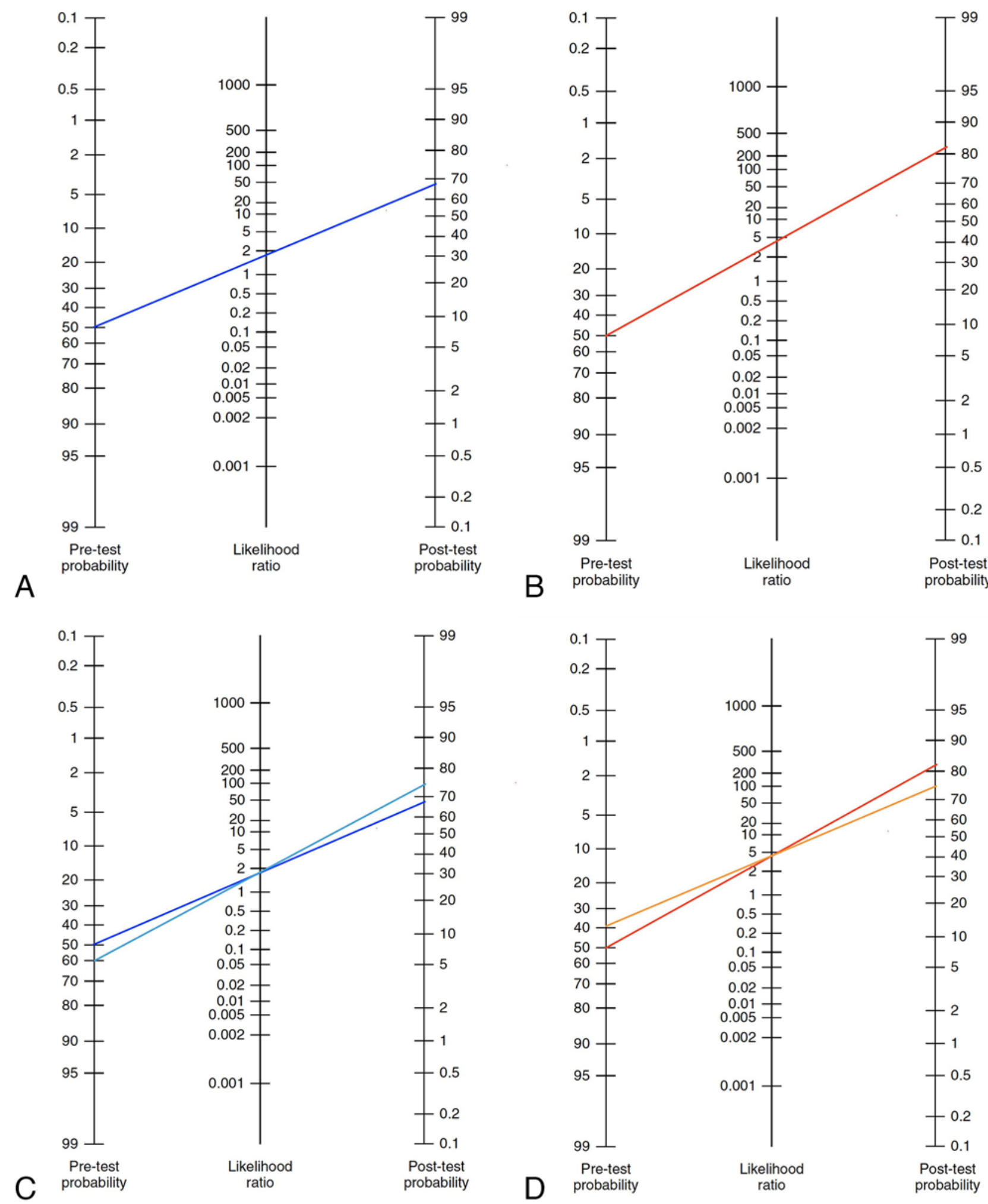

FIG. 3. Bayes' nomogram as applied to clinical decision-making. A: TBM with $50 \%$ pretest probability and likelihood ratio of 1.89 on CSF analysis. B: SAH with $50 \%$ pretest probability and likelihood ratio of 4.03 on CSF analysis. C: Readjusted pretest probability for TBM, which now presents a relatively higher posterior probability (cyan line). D: Readjusted pretest probability for SAH showing relatively lower posterior probability (orange line) than TBM. Nomogram based on information provided in Fagan TJ. Letter: Nomogram for Bayes theorem. N Engl J Med. 1975;293(5):257.45 
Consequently, the posterior probability of TBM becomes relatively higher (cyan-colored line in Fig. 3C) compared with that of SAH (orange line in Fig. 3D).

The degree of posterior probability then informs the subsequent management. When considering SAH for this patient, the neurosurgeon should not offer any treatment without the benefit of additional tests that would increase the likelihood ratio, especially given that the available treatments for a ruptured aneurysm are not without risks. By contrast, for TBM, the clinician only relies on the discriminative clinical and laboratory features, because the yields of cultures and polymerase chain reaction-based diagnostics are often low. ${ }^{39,42,43}$ From the foregoing example, it then becomes justified to reach the treatment threshold ${ }^{44}$ for the initiation of antituberculosis medication despite some degree of uncertainty that remains, ${ }^{42}$ and to advise constant follow-up through telemedicine to monitor disease resolution or possible development of shunt-requiring hydrocephalus.

\section{Conclusions}

Brought about by the demands of the pandemic and given the limitations in a low-resource setting, we offered our patients a telemedicine setup. Subsequent evidence showed that this setup, albeit imperfect, would benefit the socioeconomically disadvantaged population in terms of satisfaction and cost savings. Through the example of an actual case, we proffered that a recapitulation of the basics of the Bayesian framework might be useful to enhance clinical judgment toward greater rigor in comprehensive history taking and nuanced clinical decision-making. Incorporating such a fundamental application to the toolkit of telemedicine becomes all the more important given the physician's responsibility to diagnose and treat patients efficiently and effectively, while not imposing undue medical cost burdens onto patients, particularly during these difficult times. We hope that this report serves as a testament to the creativity and resiliency of neurosurgeons in imagining new ways of being responsive to the needs of our vulnerable patients, especially those in less fortunate parts of the world.

\section{Acknowledgments}

We would like to thank Drs. John Emmanuel Y. Custodio, Oliver Ryan M. Malilay, Kirby C. Manigos, Eric Paolo M. Palabyab, Tito Guillermo D. Rejante, Katrina F. Villanueva, and Llarx Saundt J. Yu for their assistance in telemedicine provision, and Dr. Antonio A. Rafael for his lifetime service in the neurosurgical center.

\section{References}

1. Jean WC, Ironside NT, Sack KD, et al. The impact of COVID-19 on neurosurgeons and the strategy for triaging non-emergent operations: a global neurosurgery study. Acta Neurochir(Wien). 2020;162(6):1229-1240.

2. Ferraris KP, Matsumura H, Wardhana DPW, et al. The state of neurosurgical training and education in East Asia: analysis and strategy development for this frontier of the world. Neurosurg Focus. 2020;48(3):E7.

3. Simko A, Han SH, Aldana PR. Telemedicine: providing access to care in pediatric neurosurgery to underserved communities. World Neurosurg. 2020;138:556-557.
4. Basil GW, Eichberg DG, Perez-Dickens M, et al. Letter: Implementation of a neurosurgery telehealth program amid the COVID-19 crisis - challenges, lessons learned, and a way forward. Neurosurgery. 2020;87(2):E260-E262.

5. Greven ACM, Rich CW, Malcolm JG, et al. Letter: Neurosurgical management of spinal pathology via telemedicine during the COVID-19 pandemic: early experience and unique challenges. Neurosurgery. 2020;87(2):E192-E196.

6. LoPresti MA, McDeavitt JT, Wade K, et al. Letter: Telemedicine in neurosurgery - a timely review. Neurosurgery. 2020; 87(2):E208-E210.

7. Blue R, Yang AI, Zhou C, et al. Telemedicine in the era of Coronavirus Disease 2019 (COVID-19): a neurosurgical perspective. World Neurosurg. 2020;139:549-557.

8. Advisory No. 3. Recommendations for Reopening of Outpatient Clinics and Elective Surgery During the COVID-19 Pandemic. Academy of Filipino Neurosurgeons. Accessed October 22, 2020. http://afninc.org/afn-advisory-no-3/

9. Isip-Tan IT, Marcelo AB, Sarmiento F, et al. Telemedicine: Guidance for Physicians in the Philippines. University of the Philippines-Manila Medical Informatics Unit; 2020. Accessed October 22, 2020. https://www.philippinemedicalassociation. org/wp-content/uploads/2020/05/1-Telemedicine-for-HealthProfessionals.pdf

10. Leckner S, Facht U. A Sampler of International Media and Communication Statistics 2010. Nordicom; 2011. Accessed October 22, 2020. https://www.nordicom.gu.se/sites/default/ files/publikationer-hela-pdf/nmt12_a_sampler_of_0.pdf

11. Philippines is world's top social media user: study. ABSCBN News. February 1, 2018. Accessed October 22, 2020. https://news.abs-cbn.com/focus/02/01/18/philippines-isworlds-top-social-media-user-study

12. Facebook: active users worldwide. Statista. Accessed June 17, 2020. https://www.statista.com/statistics/264810/number-ofmonthly-active-facebook-users-worldwide/

13. Number of Facebook users in the Philippines from 2017 to 2019 with a forecast until 2025. Statista. Accessed June 17, 2020. https://www.statista.com/statistics/490455/number-ofphilippines-facebook-users/

14. Smith WR, Atala AJ, Terlecki RP, et al. Implementation guide for rapid integration of an outpatient telemedicine program during the COVID-19 pandemic. J Am Coll Surg. 2020; 231(2):216-222.e2.

15. Notification of enforcement discretion for telehealth From US Department of Health \& Human Services. HHS.gov. Accessed October 22, 2020. https://www.hhs.gov/hipaa/ for-professionals/special-topics/emergency-preparedness/ notification-enforcement-discretion-telehealth/index.html

16. Institute of Medicine Committee on Quality of Health Care in America. Crossing the Quality Chasm: A New Health System for the 21st Century. National Academies Press; 2001.

17. Meara JG, Leather AJM, Hagander L, et al. Global Surgery 2030: evidence and solutions for achieving health, welfare, and economic development. Lancet. 2015;386(9993):569624.

18. Schieber GJ, Gottret P, Fleisher LK, Leive AA. Financing global health: mission unaccomplished. Health Aff (Millwood). 2007;26(4):921-934.

19. 2018 Global Reference List of 100 Core Health Indicators (plus health-related SDGs). World Health Organization; 2018. Accessed October 22, 2020. http://www.who.int/ healthinfo/indicators/2018/en/

20. Brent RJ. Cost-Benefit Analysis and Health Care Evaluations. Edward Elgar Publishing Ltd; 2003.

21. Glasziou P. Which methods for bedside Bayes? Evid Based Med. 2001;6(6):164-166.

22. Xu K, Evans DB, Kawabata K, et al. Household catastrophic health expenditure: a multicountry analysis. Lancet. 2003; 362(9378):111-117. 
23. Global Monitoring Report on Financial Protection in Health 2019. World Health Organization and the International Bank for Reconstruction and Development/The World Bank; 2019. Accessed October 22, 2020. https://www.who.int/healthinfo/ universal_health_coverage/report/fp_gmr_2019.pdf

24. Updated Full Year 2018 Official Poverty Statistics of the Philippines. Philippine Statistics Authority; 2020. Accessed October 22, 2020. https://psa.gov.ph/sites/default/ files $/ 2018 \% 20$ Official\%20Poverty\%20Statistics\%20v1_ June $\% 2004 \% 2 C \% 202020 . p d f$

25. Servadei F, Rossini Z, Nicolosi F, et al. The role of neurosurgery in countries with limited facilities: facts and challenges. World Neurosurg. 2018;112:315-321.

26. Thakar S, Rajagopal N, Mani S, et al. Comparison of telemedicine with in-person care for follow-up after elective neurosurgery: results of a cost-effectiveness analysis of 1200 patients using patient-perceived utility scores. Neurosurg Focus. 2018;44(5):E17.

27. Dadlani R, Mani S, Ganesh AU J, et al. The impact of telemedicine in the postoperative care of the neurosurgery patient in an outpatient clinic: a unique perspective of this valuable resource in the developing world-an experience of more than 3000 teleconsultations. World Neurosurg. 2014; 82(3-4):270-283.

28. Bergmo TS. Can economic evaluation in telemedicine be trusted? A systematic review of the literature. Cost Eff Resour Alloc. 2009;7:18.

29. Ahmed SN, Mann C, Sinclair DB, et al. Feasibility of epilepsy follow-up care through telemedicine: a pilot study on the patient's perspective. Epilepsia. 2008;49(4):573-585.

30. Snyder SR. Editorial. Telemedicine for elective neurosurgical routine follow-up care: a promising patient-centered and cost-effective alternative to in-person clinic visits. Neurosurg Focus. 2018;44(5):E18.

31. Gleason PM, Harris JE. The bayesian approach to decision making and analysis in nutrition research and practice. $J$ Acad Nutr Diet. 2019;119(12):1993-2003.

32. Gill CJ, Sabin L, Schmid CH. Why clinicians are natural bayesians. BMJ. 2005;330(7499):1080-1083.

33. Hall GH. The clinical application of Bayes' theorem. Lancet. 1967;2(7515):555-557.

34. Wolf FM, Gruppen LD, Billi JE. Differential diagnosis and the competing-hypotheses heuristic. A practical approach to judgment under uncertainty and Bayesian probability. JAMA. 1985;253(19):2858-2862.

35. Perry JJ, Stiell IG, Sivilotti MLA, et al. Clinical decision rules to rule out subarachnoid hemorrhage for acute headache. JAMA. 2013;310(12):1248-1255.

36. Weir B. Headaches from aneurysms. Cephalalgia. 1994; 14(2):79-87.

37. Tsao TC, Tsai YH, Lan RS, et al. Fever characteristics in tuberculosis-clinical observation in 597 cases. Changgeng $Y i$ Xue Za Zhi. 1989;12(2):81-88.
38. Ragonnet R, Trauer JM, Geard N, et al. Profiling Mycobacterium tuberculosis transmission and the resulting disease burden in the five highest tuberculosis burden countries. BMC Med. 2019;17(1):208.

39. Thwaites GE, van Toorn R, Schoeman J. Tuberculous meningitis: more questions, still too few answers. Lancet Neurol. 2013;12(10):999-1010.

40. Foppiano Palacios C, Saleeb PG. Challenges in the diagnosis of tuberculous meningitis. J Clin Tuberc Other Mycobact Dis. 2020;20:100164.

41. Wood MJ, Dimeski G, Nowitzke AM. CSF spectrophotometry in the diagnosis and exclusion of spontaneous subarachnoid haemorrhage. J Clin Neurosci. 2005;12(2):142-146.

42. Pasco PM. Diagnostic features of tuberculous meningitis: a cross-sectional study. BMC Res Notes. 2012;5:49.

43. Thwaites GE, Chau TTH, Stepniewska K, et al. Diagnosis of adult tuberculous meningitis by use of clinical and laboratory features. Lancet. 2002;360(9342):1287-1292.

44. Connors GR, Siner JM. Clinical reasoning and risk in the intensive care unit. Clin Chest Med. 2015;36(3):449-459.

45. Fagan TJ. Letter: Nomogram for Bayes theorem. $N$ Engl J Med. 1975;293(5):257.

\section{Disclosures}

The authors report no conflict of interest concerning the materials or methods used in this study or the findings specified in this paper.

\section{Author Contributions}

Conception and design: Ferraris. Acquisition of data: Ferraris, Golidtum. Analysis and interpretation of data: Ferraris, Zuñiga, Bautista. Drafting the article: Ferraris, Golidtum. Critically revising the article: Ferraris, Bautista, Alcazaren, Seng, Navarro. Reviewed submitted version of manuscript: Ferraris, Golidtum, Bautista. Approved the final version of the manuscript on behalf of all authors: Ferraris. Statistical analysis: Ferraris, Golidtum, Zuñiga, Bautista. Administrative/technical/material support: Ferraris, Golidtum, Zuñiga, Alcazaren, Seng, Navarro. Study supervision: Ferraris, Alcazaren, Seng, Navarro.

\section{Correspondence}

Kevin Paul Ferraris: Jose R. Reyes Memorial Medical Center, Manila, Philippines.kpferraris@gmail.com. 